\title{
Chemical Pathology
}

\section{GREEN LABS}

Tony Badrick

RCPA Quality Assurance Programs, St Leonards, NSW, Australia

McAlister et al. ${ }^{1}$ in the MJA recently described the carbon footprint of an Australian clinical laboratory. The article was important as it highlighted the importance of healthcare organisations becoming aware of their environmental impact.

Pathology organisations, like every corporate citizen, need be aware of their environmental responsibility. There is a literature on how laboratories can reduce their impact ${ }^{2}$ by developing an Environmental Management System (EMS). ${ }^{3}$

Benefits to an organisation of implementing an include improved environmental awareness and community relationships, cost savings and business efficiencies, regulatory compliance, improved marketing opportunities and corporate image and reduced risk of disaster.

To implement an EMS, an organised approach is essential in order to identify and manage environmental impacts and communicate and foster awareness and responsible environmental behaviour among key stakeholders including staff. Rather than just establishing an environmental policy, undertaking certification to an EMS standard can provide a framework for the adoption of key activities relating to environmental aspects in an organisation, increase accountability for those stakeholders and imbed continuous improvement across the organisation.

\section{References}

1. McAlister S, Barratt AL, Bell KJ, et al. The carbon footprint of pathology testing. Med J Aust 2020; 212: 377-82.

2. Lopez J, Badrick T. Proposals for the mitigation of the environmental impact of clinical laboratories. Clin Chem Lab Med 2012; 50: 1559-64.

3. International Organisation for Standardisation. ISO 14001: 2015 Environmental Management Systems - Requirements with Guidance for Use. Sep 2015; cited 9 Dec 2020. https:// www.iso.org/standard/60857.html

\section{SERUM FREE LIGHT CHAINS}

Karl W. Baumgart

Immunology, Douglass Hanly Moir Pathology, Sonic

Healthcare, NSW, Australia

Serum free light chain estimations entered most clinical laboratory test menus from September 2008. Their main clinical utility is in the identification of persons who might have a light chain only plasma cell dyscrasia. Other reasons clinicians request these assays are for the monitoring of persons with myeloma and monitoring progression of renal impairment. Increased sensitivity for detection of excessive free light chain production over urine protein electrophoresis and immunofixation may earlier identify persons with AL amyloid or myeloma. Non-compliance by patients with requests for urine EPG-IFE can be offset by serum free light chain estimations.
Serum free light chain assays are unable to define clonality in a person with abnormal free light chain levels unlike serum or urine protein electrophoresis. Measurements can be insensitive to prozone effects, potentially underestimating light chain levels despite careful testing strategies. Some monoclonal free light chain bands may have idiosyncratic charge and other physical characteristics that can frustrate good assay systems. Physician responses to abnormal results may vary according to clinical context in terms of known monoclonal bands, renal impairment, suspicion of AL amyloid, immunosuppressive treatments. Robust and reliable serum free light chains have earned a mature role in serum protein studies with strong support by clinician users.

\section{GENETIC TESTING FOR FAMILIAL HYPERCHOLESTEROLAEMIA}

John R. Burnett ${ }^{1,2}$

${ }^{1}$ Department of Clinical Biochemistry, PathWest Laboratory Medicine, Royal Perth Hospital and Fiona Stanley Hospital Network, Perth, WA, Australia; and ${ }^{2}$ School of Medicine, University of Western Australia, Nedlands, WA, Australia

Familial hypercholesterolaemia $(\mathrm{FH})$ is a codominant genetic disorder causing premature atherosclerotic cardiovascular disease (ASCVD). It is common, affecting 1 in 250 individuals worldwide and of the $\sim 100,000$ Australians estimated to have FH, most are undiagnosed and treatment is often suboptimal. Several scoring algorithms based on low-density lipoprotein-cholesterol (LDL-C) levels, physical findings, and elements of personal and family history, help clinicians diagnose $\mathrm{FH}$ at varying levels of confidence ranging from possible to definite. Targeted DNA sequencing to identify the underlying pathogenic variant in any of the three FH genes ( $L D L R, A P O B$, and PCSK9) has become more accessible, but it is not essential for diagnosis of $\mathrm{FH}$, and is currently not widely used. However, identification of a pathogenic variant is considered the 'gold standard' for FH diagnosis. Expanded FH panels can identify pathogenic variants in other hypercholesterolaemia genes, such as APOE, LIPA, and ABCG5/ 8. FH cascade screening is recommended to identify affected family members, and the benefits of early intervention to lower LDL-C and reduce the associated ASCVD burden are clear. National registries can also play a key role in identifying patients with FH. FH genetic testing should be incorporated as standard of care for patients and their relatives with suspected FH.

\section{THE PROBLEM(S) WITH TROPONIN}

Christina Trambas ${ }^{1,2}$

${ }^{1}$ St Vincent's Pathology, St Vincent's Hospital, Melbourne, Vic, Australia; and ${ }^{2}$ Austin Pathology, Austin Health, Heidelberg, Vic, Australia

Cardiac troponin has long been the preferred biomarker for the evaluation of acute coronary syndrome. Successive improvements 